\section{DYING MATTERS SO WHY DON'T WE TALK ABOUT IT: A SURVEY OF STAFF AND VOLUNTEERS IN HOME, HOSPICE AND HOSPITAL SETTINGS IN SOMERSET UK}

Janet Gillett, Melody Schultz, Lucy Nicholls, Charlie Davis. Taunton and Somerset NHS Foundation Trust, Yeovil District Hospital NHS Foundation Trust, Somerset Partnership NHS Foundation Trust, St Margarets Somerset Hospice, Dorothy House Hospice

\subsection{6/spcare-2020-PCC.42}

Background Discomfort talking about death and dying inhibits advance care planning (ACP) conversations. This survey aimed to raise awareness of end of life care (eolc) across home (community), hospices and NHS hospitals in Somerset and to explore staff and volunteers' views about talking about dying. Respondents were encouraged to think beyond the patient-clinician relationship and consider how they interact with their families and communities when considering eolc.

Methods Staff and volunteers working in 2 district general hospitals, community services and 2 hospices were invited to complete an online survey during Dying Matters week (May 2019). Volunteers also encouraged completion of the survey on an ipad. The survey questions explored: most common worries for people at eol; attitudes towards talking about death and dying with other people (colleagues/patients/own family/friends); how much was known about ACP; perceived education needs. Free text data was extracted into word clouds and 10 point linear scale responses analysed using excel.

Results $58 \%$ of 1045 respondents were staff with clinical responsibilities. The 3 most frequently-used words around worries at eol were: family, pain, leaving. $20 \%$ were not comfortable talking with another person about dying, with similar distribution of responses from clinical and non clinical respondents. They were all more comfortable talking with colleagues. $44 \%$ of respondents said they knew little or nothing about ACP with non clinical respondents having less knowledge. More than half wanted training to develop skills to talk about dying, including $61 \%$ nurses and doctors.

Conclusions Care of the dying is not the exclusive domain of clinical staff. More dying people need to have opportunity to talk about what is important to them and their future. Empowering people through education and training (clinical/ non-clinical/staff/volunteers) in their 'outside-work' lives to have conversations about 'the future' is fundamental to achieving sustainable quality improvements in eolc.

\section{OPIOID PRESCRIPTIONS AND PATIENT UNDERSTANDING OF OPIOIDS WITHIN AN ACUTE HOSPITAL SETTING}

Peter Goodwin, Mark Banting. University of Southampton

\subsection{6/spcare-2020-PCC.43}

Introduction There are guidelines on the prescription of opioids relating to choice in renal impairment and the ratio of modified release to immediate release. When prescribing opioids, it is essential patients understand what they are, how to take them, and they are counselled on side effects and limitations. Staff must also understand prescribing and counselling regarding opioids.
Method 2 part study:

1. Service Evaluation - Questionnaires filled in by both staff and patients, regarding their prescribing, knowledge and perception of opioids.

2. Audit - Comparing whether patients are prescribed the correct opioid in relation to their renal function, and whether the ratio of modified release opioid to immediate release is correct.

Results At the time of writing, we had 36 patient and 10 staff responses to the questionnaires, as well as 45 recordings of patients' MR:PRN ratio. We had reviewed approximately 700 prescriptions of opioids to compare with renal function.

Service Evaluation: $80 \%$ of staff said they never provided opioid information leaflets to patients, and many staff mentioned fear associated with opioid dependence and terminal illness when prescribing. $22.2 \%$ of patients could not name the opioid they were receiving, and only $16.7 \%$ said they had received the opioid information leaflet.

Audit: $30 \%$ of patients with an eGFR $<30$ had been prescribed morphine (in contrast to guidelines). 67\% patients had an incorrect MR:PRN ratio.

Conclusion and further work Staff showed good competency levels of opioid understanding and prescriptions but many patients had not been given adequate information regarding opioids. The audit shows reasonable prescribing of opioids in renal impairment however need for education about MR:PRN ratios. We aim to further educate staff about opioids and their prescription and also trial a full roll out of our patient leaflet 'strong opioids for cancer pain' re-auditing its use over the next year.

\section{FROM AN INTENSIVE CARE UNIT (ICU) TO A HOSPICE; A CASE STUDY HIGHLIGHTING THE NEED FOR TAILORED STAFF EDUCATION IN RESPONSE TO THE INCREASINGLY COMPLEX CASE-MIX IN SPECIALIST PALLIATIVE CARE}

A Grundy, V Ewart, D Wakefield. Marie Curie Hospice

\subsection{6/spcare-2020-PCC.44}

Background Palliative Medicine has expanded rapidly as a specialty to encompass non-cancer patients, with multiple co-morbidities, in a wider range of settings. Adaptation of existing services with ongoing staff training is essential to meet the evolving demographics and demand.

Case report An 81-year-old diabetic patient with peripheral vascular disease developed necrosis of the foot. Revascularisation attempts were complicated leading to ICU admission for intravenous antibiotics and haemodynamic support. They were diagnosed with necrotising fasciitis but were not fit for further surgery. Referral was made from ICU to hospice, their preferred place of death. After inotrope withdrawal, the patient was transferred with a bupivacaine spinal line in-situ for pain control. Diabetic control was challenging in the context of variable oral intake and sepsis. Morphine and midazolam syringe drivers were titrated; the patient died peacefully eight days after admission.

Evolving case mix: Admissions to the hospice inpatient unit from 2015-2019 were reviewed retrospectively. Although we were unable to identify those patients transferred directly from ICU to hospice, the proportion of patients admitted to 
the hospice with non-malignant conditions had risen from $11 \%$ (2015) to $21 \%$ (2018) during this time.

Staff training Teaching sessions were provided for staff to highlight key considerations when caring for patients with necrotising fasciitis, including symptoms and infection control advice. Feedback was gathered from staff attending teaching with $82 \%$ rating it as 'useful' or 'very useful' and $90 \%$ indicating that they would be interested in future education sessions.

Conclusion The breadth of patients referred for inpatient hospice management is growing. The case outlined may represent an emerging patient subgroup; those without a prior palliative diagnosis whereby active treatment of an acute complication has failed. Teaching sessions proved beneficial to hospice staff and should be considered in the future to promote individualised integration of care across disciplines.

\section{IMPROVING COMMUNICATION AND DOCUMENTATION OF END OF LIFE CONVERSATIONS, A SIMULATION BASED PROJECT}

E Haire, S Woods, N Wiggins. Great Western NHS Foundation Trust

10.1136/spcare-2020-PCC.45

Approximately $89.6 \%$ of patients' who are going to die in the next 12 months are admitted to hospital at least once. Evidence shows that good communication reduces hospital stays through advance care planning in the last 12 months of life. End of Life and DNAR discussions are part of the F2 curriculum and the development of good communication skills is a central part of clinical training. It is important to note that it is now a legal requirement that doctors involve patients and families in decisions regarding treatment escalation and resuscitation following the Tracey Judgement.

A recent audit undertaken at our Trust showed that there is scope for improving the quality of treatment escalation conversations the doctors are having with patients and their families as well as the documentation of these discussions.

An initial survey of F2 doctors showed that 92\% had previously had TEP discussions with a patient, $77 \%$ with a relative and $69 \%$ had completed a TEP form. 46\% reported not feeling confident having these discussions with main concerns not knowing what to say to initiate the conversation, being unable to answer questions and causing distress to both the patients and their family

A SIM course was delivered to improve the communication skills and confidence of F2 doctors allowing them to practice with actors in a safe learning environment. This learning was consolidated by debriefing with consultants in geriatric and palliative medicine. Post course feedback showed all participants enjoyed the course, $90 \%$ found it useful for clinical practice and $80 \%$ would recommend it to their colleagues. Significantly, all participants feel confident in discussing TEP following the SIM and commented on the detailed feedback.

This should lead to an increase in the number of patients who have these documented discussions which we know improves patient care.

\section{A MIXED-METHODS STUDY TO INVESTIGATE NURSING ATTITUDES TOWARDS ADMINISTRATION OF 'AS REQUIRED' PRESCRIBED SYMPTOM CONTROL MEDICATION AT END OF LIFE IN A HOSPITAL SETTING}

Elke Hall, Fiona Dakin, Christine Hirsch, John Speakman, Jon Tomas. University of Birmingham, University Hospital Birmingham NHS Trust

10.1136/spcare-2020-PCC.46

Background Anticipatory drugs for symptom control in dying patients are commonly prescribed in the acute hospital setting. The decision to administer 'as required' drugs usually lie with the ward nursing team who may have varied experience in end of life care. A previous survey informed changes to end of life care training offered within the Trust. This prompted the team to undertake a follow-up survey to determine whether the adapted training provision was meeting the needs of nursing staff administering 'as required' drugs at the end of life and to aid understanding of nurse decision making in this area of drug administration in the hospital setting.

Methods A questionnaire based on the previous survey was developed and piloted. A range of question styles were used including Likert scale, two ranking scales based on scenarios, and free text responses. Ethical approval was gained. Hard copies of the questionnaire were distributed to nurses on wards with the highest death rates together with elderly care wards. 100 copies of the questionnaire were distributed. Completed anonymous responses were coded and statistically analysed using SPSS 26.

Results The response rate was 62\%. Just under 50\% respondents reported being 'very confident' in recognising symptoms at the end of life. This was similar across the wards surveyed (oncology, acute medical, elderly care). Scenario ranking questions based on treatment of pain and agitation resulted in appropriate responses. 39\% of respondents had undertaken the Trust palliative care training. Five respondents (8\%) expressed fears around administration of medication at end of life.

Conclusion Most hospital nurses reported confidence in recognising end of life symptoms. Preliminary results have been shared with the specialist team, awaiting the final report to inform further development of training to improve the confidence and decision making of newly qualified nurses in this area of drug administration.

\section{PSYCHIATRY \& PALLIATIVE CARE: MEETING INTER-PROFESSIONAL, MULTI-DISCIPLINARY EDUCATIONAL NEEDS}

Daniel Hughes, Sarah Yardley, Katie Weatherstone. Central and North West London NHS Foundation Trust

\subsection{6/spcare-2020-PCC.47}

Introduction There is increasing evidence of higher rates of psychiatric disorder in palliative care patients. Navigating between palliative and psychiatric services can be challenging, and links between these specialities need to be strengthened to improve patient care and develop cross-disciplinary learning. Appropriate educational and networking opportunities for professionals to explore these issues has been limited. 